Mareque Álvarez-Santullano, M. y De Prada Creo, E. (2018). Evaluación de las competencias profesionales a través de las prácticas externas: incidencia de la creatividad. Revista de Investigación Educativa, 36(1), 203-219.

DOI: http://dx.doi.org/10.6018/rie.36.1.275651

\title{
Evaluación de las competencias profesionales a través de las prácticas externas: incidencia de la creatividad
}

\author{
Evaluation of professional competencies through external \\ internships: the effect of creativity
}

\author{
Mercedes Mareque Álvarez-Santullano y Elena De Prada Creo \\ Universidad de Vigo
}

\begin{abstract}
Resumen
Esta investigación pretende analizar en qué medida existe una correspondencia entre la formación académica recibida por el alumnado y las exigencias del mercado laboral desde el punto de vista competencial, con especial atención a la creatividad. Para alcanzar este objetivo se ha recurrido a la información obtenida de las prácticas externas en empresas, que proporcionan un entorno ideal para el desarrollo y evaluación de las competencias profesionales que debe adquirir el alumnado, ya que constituyen un elemento de transición para la incorporación al mercado laboral. Se han examinado un total de 274 prácticas realizadas durante los cursos 2013-14 y 2014-15 de los estudios de grado de la Facultad de Ciencias Empresariales y Turismo de la Universidad de Vigo. Tanto los alumnos como los tutores en las empresas recibieron un informe para valorar, de forma individual, una relación de idénticas competencias para ambos grupos desarrolladas a lo largo de las prácticas. Los resultados obtenidos en nuestro estudio muestran que, en general, la puntuación promedio de todas las competencias evaluadas, tanto por alumnos como por los tutores de las empresas, es, en todos los casos, alta, exceptuando la creatividad, competencia que ha sido valorada con la puntuación más baja por ambos grupos.

Palabras clave: practicum; competencias profesionales; creatividad; empleabilidad.
\end{abstract}

Correspondencia: Mercedes Mareque Álvarez-Santullano, chedesmareque@uvigo.es, Facultad de Ciencias Empresariales y Turismo. Campus Universitario - 32004 Ourense.

Grupo investigación: RGEAF Economics and Business Administration for Society (ECOBAS), Universidad de Vigo. 


\begin{abstract}
The aim of this research study is to analyse to what extent the academic training received by students and the requirements of the job market are related from a competence point of view, with special attention paid to creativity. To meet this goal, the information obtained from the external internships in companies has been a valuable source that provides an ideal context for the development and assessment of the professional competences that students need to acquire, as they constitute a transitional stage for their incorporation into the labour market. 274 internships carried out during the academic years 2013-14 and 2014-15 were analysed, these internships forming part of the degree studies at the Faculty of Business Studies and Tourism at the University of Vigo. Both students and company tutors were given a form to individually assess a list of identical competences for both groups, presumably acquired or developed during their internships. The results of the study reveal that, in general, the average score of all the evaluated competences given by both students and tutors is high, except for creativity, a competence that was scored as the lowest by both groups.

Keywords: practicum, job skills, creativity, employment.
\end{abstract}

\title{
Introducción
}

Este trabajo parte de la preocupación por la calidad educativa a nivel universitario y cómo ésta se traduce en la incorporación al mercado laboral del alumnado. Si bien la evaluación de las materias tiene un reflejo directo en las calificaciones otorgadas por los profesores y poseemos datos sobre si esos conocimientos han sido o no asimilados, resulta mucho más difícil evaluar las competencias adquiridas durante la titulación, competencias que van a ser determinantes en la práctica profesional de los egresados.

Si todas las competencias a evaluar son relevantes para este objetivo, especial interés suscita la creatividad, fundamental para la innovación y el emprendimiento inherentes a las titulaciones objeto de estudio, la cual hemos constatado como la gran ausente, ya que aproximadamente la mitad de las universidades públicas españolas no la mencionan en sus memorias, pese a las abrumadoras evidencias de su importancia en el mundo empresarial y las recomendaciones hechas desde Europa sobre su relevancia como competencia transversal.

El Marco de Referencia Europeo recomienda específicamente la creatividad como uno de los temas sobre los que se han de articular todas las competencias y, por tanto, la sitúa como un elemento de gran importancia para la formación competencial global del alumno. Pero esta recomendación no se queda sólo en un plano teórico a nivel institucional sino que es la principal demanda de las empresas en la actualidad, que ven el binomio creatividad-innovación como clave para hacer frente a la crisis económica (Ghosh, 2014), destacándose que no sólo ha de restringirse a los puestos ejecutivos tradicionalmente asociados con la creatividad sino hacerla extensiva a todos los trabajadores (Zhou, 2007) y a todo tipo de empresas (Epstein, Kaminaka, Phan \& Uda, 2013).

En la ordenación de las enseñanzas universitarias oficiales, introducida por exigencias del proceso de construcción del Espacio Europeo de Educación Superior (en 
adelante EEES), se puso un especial énfasis en la realización de prácticas externas por los estudiantes universitarios, previendo que los planes de estudios de grado contuviesen toda la formación teórica y práctica que el estudiante debía adquirir. En este mismo sentido, el Estatuto del Estudiante Universitario, reconoce el derecho de los estudiantes de Grado a disponer de la posibilidad de realización de prácticas, curriculares o extracurriculares, que podrán realizarse en entidades externas y en los centros, estructuras o servicios de la Universidad, según la modalidad prevista, y garantizando que sirvan a la finalidad formativa de las mismas (Real Decreto 592/2014).

El complejo y cambiante entorno al que tendrán que enfrentarse nuestros alumnos universitarios, futuros profesionales, les llevará a tener que demostrar que durante sus estudios han adquirido una formación integral que hace que la teoría y la práctica se encuentren perfectamente interrelacionadas, y que, por tanto, no solo posean los conocimientos académicos y técnicos adecuados, sino que también hayan adquirido habilidades profesionales, actitudes y valores que manifiesten su capacidad de enfrentarse a un mercado laboral que cada vez es más competitivo. A este conjunto de capacidades, habilidades y actitudes complementarias a la formación técnica que los alumnos deben adquirir se les ha denominado competencias profesionales (Biedma, Gómez Aguilar \& Ruiz Barbadillo, 2011) ${ }^{1}$.

Las prácticas externas servirán como complemento de la formación teórico-práctica recibida en la universidad y, constituirán un espacio preprofesional integrado por actividades dirigidas a capacitar profesionalmente a los alumnos (Molina, Bolivar \& Burgos, 2007).

Uno de los objetivos de todos los estudios superiores es preparar al alumnado para su integración en el mundo laboral y profesional. El sistema universitario tiene un importante papel relacionado con la enseñanza de competencias a los alumnos, con la finalidad de que estos futuros profesionales obtengan las habilidades prácticas necesarias que les capacitarán para ejercer de forma óptima una labor profesional. El EEES, conocedor de esta necesidad, da especial relevancia a que los estudios se orienten hacia la empleabilidad, desarrollando competencias profesionales $\mathrm{y}$, habilitando y capacitando a los alumnos para el desarrollo de actividades de carácter profesional. Este compromiso de empleabilidad asumido por el EEES viene reflejado mediante la posibilidad de que los alumnos puedan realizar durante el periodo formativo prácticas externas, completando su formación (García Manjón \& Pérez López, 2008). Si bien, como señalan González Lorente y Martínez Clares (2016) existen una serie de factores que inciden en el proceso de inserción laboral, el denominado triángulo

1 La Ley Orgánica 5/2002 de 19 de junio, de las Cualificaciones y de la Formación Profesional entiende por competencia profesional el conjunto de conocimientos y capacidades que permiten el ejercicio de la actividad profesional conforme a las exigencias de la producción y el empleo. Por el contrario, el Libro Blanco del Título de Grado en Economía y en Empresa (Agencia Nacional de Evaluación de la Calidad y Acreditación, ANECA, 2005) define este concepto exclusivamente como una competencia específica ligada al saber hacer, distinguiéndolo así de los conocimientos disciplinares que aportan el saber y de las competencias académicas. Este concepto ha sido ampliamente definido, puede consultarse: Boyatzis, 1982; Climént, 2010; Delgado, Borges Bravo, García Albero, Oliver Cuello y Salomón Sancho, 2005; Fernández, 2005; García Manjón y Pérez López, 2008; Hartog, 1992; Jurado, 2009; Martínez Ruiz y Sauleda, 2005; Mertens, 1996; Oliveros, 2006. 
básico formación-orientación-empleo. Es decir, el éxito de los estudiantes a la hora de acceder a un empleo, no sólo depende de la formación recibida, sino también de sus circunstancias y actitudes, así como del contexto laboral, social y económico en el que se encuentran y del estado del mercado de trabajo, entre otros factores.

En el diseño de los actuales grados universitarios se tienen en cuenta no solo competencias específicas -conjunto de conocimientos relevantes para cada tipo de trabajo- sino también las competencias genéricas o transversales -conducta asociada al desarrollo, comunes a las distintas ocupaciones o ramas de actividad- (Solanes, Núñez Núñez \& Rodríguez Marín, 2008). Dentro de estas competencias, la creatividad ha sido señalada como una de las de mayor relevancia en el mundo empresarial, llegando a ser, en numerosos casos, el rasgo más demandado.

Las prácticas externas deben tratar de coordinar o aunar dos funciones básicas, una que proviene del sistema educativo, la transmisión de conocimientos y desarrollo de capacidades propias del individuo, y la otra que proviene del sistema laboral, emplear los conocimientos del sistema educativo y combinarlos con la experiencia profesional y con la formación continua con el objetivo de desarrollar las competencias que conlleven un correcto desempeño del puesto de trabajo (Tejada \& Navío, 2005).

La introducción de la posibilidad de realización de prácticas externas a lo largo de los estudios universitarios reforzará la formación de los estudiantes, y les permitirá por un lado, aplicar las capacidades y conocimientos adquiridos en sus estudios, desarrollando así las competencias profesionales en el ámbito laboral, y por otro, reforzará la adquisición de conocimientos, habilidades profesionales y actitudes, demostrando sus capacidades y sus competencias. A las empresas, les permitirá comprobar si las competencias adquiridas por el alumnado, como resultado de su formación, se adaptan a las requeridas en los puestos de trabajo. Por tanto, la realización de prácticas externas en empresas proporciona un entorno ideal para el desarrollo y evaluación de las competencias profesionales que deben poseer los alumnos. La información que aporte el análisis de los resultados será de gran importancia para conocer en qué medida la formación del alumnado se adecúa a las demandas del mercado laboral. Además, debemos tener en cuenta que las empresas en sus procesos de selección están valorando cada vez más la tenencia por parte de los candidatos de competencias profesionales.

Es importante que las competencias demandadas por las empresas y las adquiridas por los estudiantes a lo largo de sus estudios universitarios sean, en la medida de lo posible, coincidentes. El análisis de las competencias profesionales en los titulados universitarios ha sido estudiado por diversos autores. Montoro, Mora y Ortiz de Urbina (2012) realizan una clasificación de estos trabajos diferenciando por un lado, entre los publicados desde un punto de vista académico (se centran en el análisis de las competencias teóricas), y por otro, los trabajos desde el punto de vista laboral, los cuales analizan las competencias prácticas. En ocasiones, es difícil separar a nivel práctico ambos tipos de competencias; así, un primer grupo de estudios analizan las competencias teóricas o del ámbito académico-educativo (Abad \& Castillo, 2004; Quintana, Raccoursier, Sánchez Guzmán, Sidler \& Toirkens, 2007), un segundo grupo, estudian la relación entre las competencias teóricas y prácticas (Biedma et al., 2011; Blanco \& Latorre, 2012; Finkel, Parra \& Roquero, 2010; Freire, Teijeiro \& Pais, 2013; 
Marzo, Pedraga \& Rivera, 2006; Montoro et al., 2012; Oliveros, 2006), y un tercero, se centran en las competencias del ámbito laboral (Gil, 2007). El trabajo que nosotros hemos desarrollado se enmarca en el segundo grupo de estudios.

\section{Método}

\section{Objetivo}

El objetivo de este trabajo es examinar en qué medida existe una correspondencia entre la formación académica recibida por el alumnado y las exigencias del mercado laboral a través del análisis de las competencias desarrolladas en las prácticas externas, con especial atención a la creatividad.

\section{Población y muestra}

La población de referencia está formada por la totalidad de las prácticas académicas externas realizadas por el alumnado durante los cursos 2013-14 y 2014-15 en la Facultad de Ciencias Empresariales y Turismo de la Universidad de Vigo que arrojó un total de 310 casos. A todos los alumnos y tutores en las empresas se les hizo entrega del correspondiente informe para que a la finalización de las prácticas realizasen la evaluación de las competencias adquiridas en las mismas y, se lo hiciesen llegar al tutor académico en la facultad. De estas 310 prácticas se recibieron los informes de evaluación de un total de 274, es decir, 548 informes, la mitad cumplimentados por los alumnos y la otra mitad por los tutores. Por tanto, la muestra objeto de estudio está formada por un total de 274 prácticas realizadas por los alumnos matriculados en las dos titulaciones de nuestro centro, Grado en Administración y Dirección de Empresas (ADE) y Grado en Turismo.

Aunque no se realiza un análisis para determinar la representatividad de la muestra, consideramos que, dado el elevado porcentaje de casos estudiados con respecto a la población total considerada (274 de 310 casos), existe un sobre muestreo que, entendemos, justifica sobradamente la representatividad de la muestra y sus resultados. En la Tabla 1 y 2 podemos apreciar la composición de la población y de la muestra por sexo y por titulación, pudiendo observar que para cualquiera de los cursos las prácticas en las dos titulaciones son realizadas en su mayoría por mujeres.

Tabla 1

Composición de la población por sexo y titulación

\begin{tabular}{lcccccc}
\hline & \multicolumn{2}{c}{ Hombres } & \multicolumn{2}{c}{ Mujeres } & \multicolumn{2}{c}{ Total } \\
\hline & No & \% & No & \% & No & \% \\
\cline { 2 - 7 } ADE & 63 & $72.41 \%$ & 118 & $52.91 \%$ & 181 & $100.00 \%$ \\
Turismo & 24 & $27.59 \%$ & 105 & $47.09 \%$ & 129 & $100.00 \%$ \\
\hline Total & 87 & $28.06 \%$ & 223 & $71.94 \%$ & 310 & $100.00 \%$ \\
\hline
\end{tabular}


Tabla 2

Composición de la muestra por sexo y titulación

\begin{tabular}{lcccccc}
\hline & \multicolumn{2}{c}{ Hombres } & \multicolumn{2}{c}{ Mujeres } & \multicolumn{2}{c}{ Total } \\
\hline \multirow{2}{*}{ ADE } & No & \% & No & \% & No & \% \\
\cline { 2 - 7 } Turismo & 54 & $33.96 \%$ & 105 & $53.30 \%$ & 159 & $100.00 \%$ \\
\hline Total & 23 & $20.00 \%$ & 92 & $46.70 \%$ & 115 & $100.00 \%$ \\
\hline
\end{tabular}

\section{Instrumento}

Los datos utilizados para la realización de nuestro trabajo se han extraído de los citados informes de valoración cubiertos por el alumno y por el tutor externo. Ambos informes, de uso obligatorio para las prácticas desarrolladas en nuestra universidad, han sido diseñados por el Área Empleo y Emprendimiento de la Universidad de Vigo los cuales contienen un conjunto de competencias a valorar tanto genéricas como específicas sobre las prácticas realizadas.

Respecto a la medición de las competencias, ambos informes contienen 7 competencias a valorar: capacidad técnica, administración de los trabajos, habilidades de comunicación oral y escrita, creatividad, iniciativa, motivación y trabajo en equipo. La medición se hace sobre una escala de 1 a 5 , donde 1 indica "mal" y 5 "excelente".

Para corroborar la fiabilidad de la escala de medida se calculó el coeficiente de Alfa de Cronbach, cuyos resultados mostraron un coeficiente alfa de .906 para la muestra de alumnos y de 0895 para la muestra de tutores, lo que implica una alta fiabilidad en ambos casos (Gliem \& Gliem, 2003; Sijtsma, 2009).

\section{Procedimiento}

Existen diversas técnicas para la identificación y evaluación de las competencias, pudiéndose distinguir entre técnicas para valorar las competencias (fundamentadas en rasgos o características de las personas), técnicas basadas en el comportamiento de las personas en sus puestos de trabajo, y técnicas que aportan valoraciones recogidas de los propios trabajadores o de otros miembros de la organización (Gil, 2007). Nuestro estudio se basa en este tercer tipo de técnicas, donde por un lado, los alumnos autoevaluarán un conjunto de competencias desarrolladas en las prácticas, y por otro, los tutores en las empresas evaluarán esas mismas competencias.

La gestión de las prácticas en la Universidad de Vigo se desarrolla bajo un proceso definido, que de forma esquemática se puede observar en la Figura 1.

- Fase 1: El alumno debe buscar y seleccionar la empresa/entidad donde va a realizar la práctica y comunicárselo al responsable de prácticas del centro.

- Fase 2: En primer lugar, el coordinador del centro valorará y decidirá si la empresa es adecuada para el desarrollo de las prácticas del alumno. Una vez 
comprobado que el departamento y funciones a desarrollar en la práctica son las adecuadas, el coordinador del centro en colaboración con la Fundación de la Universidad ${ }^{2}$ comprobará si existe convenio de colaboración con la entidad firmado y, caso de que no exista se procederá a la gestión y firma del mismo.

- Fase 3: Se procede a la tramitación y firma de la documentación de formalización de la práctica, en la cual constará toda la información de la misma: datos de la empresa, datos del alumno, duración en horas de la práctica, fecha de inicio y finalización, remuneración, departamento y funciones a desarrollar. Además, el coordinador del centro asignará al alumno un tutor interno o académico y un tutor externo o tutor en la empresa/entidad. Los tutores internos son profesores pertenecientes a la titulación en la cual cursan estudios los alumnos y son los encargados de velar por el normal desarrollo de la práctica garantizando entre otras la compatibilidad de horarios con las obligaciones académicas, formativas y de representación y participación del estudiante, así como del seguimiento efectivo de las prácticas en coordinación con el tutor externo o tutor en la entidad. Por último, en esta fase se le hace entrega tanto al alumno como al tutor externo de un informe con un conjunto de competencias desarrolladas en la práctica, las cuales deben valorar a finalización de la práctica. Estos informes una vez cubiertos serán entregados al tutor académico a finalización de la práctica.

- Fase 4: Se trata del desarrollo efectivo de la práctica, donde tanto por el tutor interno como del externo estarán a disposición del alumno para su asesoramiento, seguimiento y guiar al alumno en su aprendizaje. Una vez finalizada la práctica el tutor interno debe evaluar y certificar la práctica, lo cual hará en base a los dos informes cubiertos por el tutor interno y por el alumno, en base a una memoria que el alumno debe elaborar sobre la práctica $\mathrm{y}$, si lo considera necesario, en base a la realización de una entrevista con el alumno. Por último, toda la documentación generada en la práctica es entregada por el tutor académico al coordinador del centro para su custodia y evaluación.

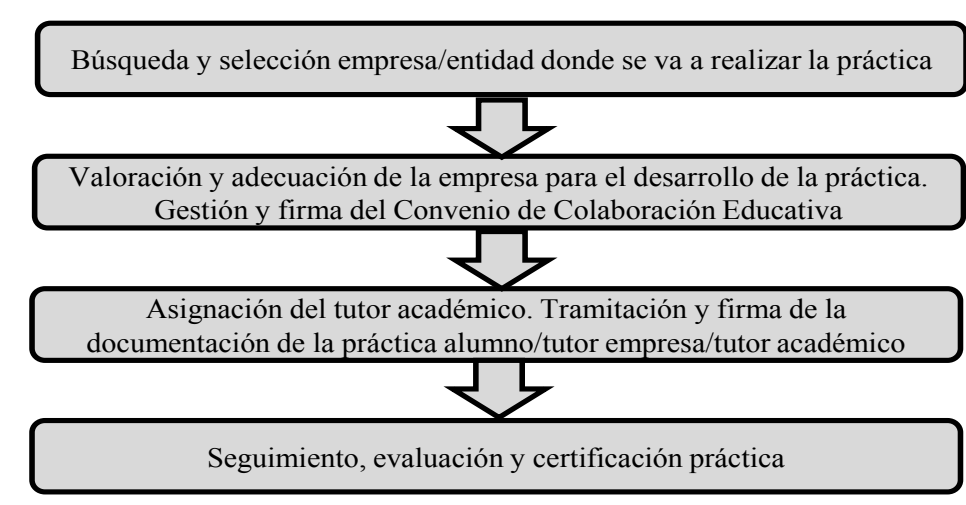

Figura 1. Proceso de prácticas.

2 Fundación Universidad de Vigo (FUVI), entidad sin ánimo de lucro creada en 1997 para impulsar actuaciones en el ámbito del empleo, del emprendimiento y de la formación. 


\section{Análisis de datos y limitaciones}

La metodología de investigación es de corte cuantitativo, fundamentalmente descriptiva, recogiendo la información aportada en los informes cubiertos por los alumnos y por los tutores en las empresas sobre las prácticas realizadas. Estos informes además de la evaluación de las competencias desarrolladas o adquiridas en las prácticas contienen otra información que nos ha permitido realizar un análisis de las mismas desde el punto de vista de género y de la titulación que cursa el alumno.

Una vez recibidos los informes por parte de los alumnos y tutores se codifican los datos utilizándose para su análisis los estadísticos descriptivos más adecuados en función de las características de los ítems. Como ya se ha comentado las competencias se han medido a través de una escala ordinal desde 1 hasta 5, donde 1 indica "mal" y 5 "excelente".

Debe tenerse en cuenta que nuestro estudio cuenta con una serie de limitaciones. Por un lado, partimos de una encuesta tipo elaborada del Área Empleo y Emprendimiento de la Universidad de Vigo y que forma parte del protocolo genérico obligatorio a seguir en la evaluación de las prácticas. En segundo lugar, dado que normalmente la empresa recibe solo un alumno el tutor en la entidad al realizar su valoración carece de un elemento comparativo para evaluar las competencias de forma simultánea, si bien si lo posee, pues cuenta con la sucesión de distintos alumnos que recibe en diferentes momentos de tiempo, ya que son muchas las empresas que cuentan con nuestro alumnado de forma sistemática y frecuente.

\section{Resultados}

A continuación vamos a presentar los resultados más relevantes de nuestro análisis sobre los ítems analizados de la muestra.

1) Competencias evaluadas por los alumnos y por los tutores en las empresas.

En relación con la importancia otorgada por los alumnos y por los tutores en las empresas a las competencias desarrolladas en las prácticas, los resultados procedentes del análisis se muestran en la Tabla 3:

- Los resultados de la media son muy aceptables en ambos cursos; así, por un lado, en todas las competencias la puntuación media dada tanto por los alumnos como por los tutores es alta y en todos los casos superior a 4.342 excepto en creatividad, donde los alumnos disminuyen hasta el 3.959. Por otro lado, comparando la puntación media dada en todas las competencias por ambos grupos, la valoración dada por los tutores siempre es superior que la de los alumnos.

- El valor de la mediana, para ambos grupos y para todas las competencias es de 5, excepto en la creatividad calificada por los alumnos que es de 4 .

- La moda, puntación más repetida por los alumnos y por los tutores es de 5.

- La desviación típica, nos indica que en todos los casos las valoraciones dadas por los alumnos tienen una mayor variabilidad que las dadas por los tutores, que son más uniformes. 
- En referencia a las puntaciones máximas y mínimas, cabe destacar que las mínimas más bajas son valoradas por los alumnos, exceptuando a la creatividad y a la iniciativa, donde también el valor mínimo (1) lo emiten los tutores.

- Para evaluar si las variables introducidas tienen o no poder discriminante, se lleva a cabo la prueba de igualdad de medias de los grupos, prueba Lambda de Wilks, donde se constata la existencia de diferencias significativas entre ambos grupos, alumnos y tutores, para todas las competencias excepto para las habilidades de comunicación oral y escrita, con un nivel de confianza del 95\% para la capacidad técnica y la administración de trabajos, y del 99\% para el resto de las competencias.

Tabla 3

Estadísticos descriptivos sobre la valoración de las competencias dadas por los alumnos y por los tutores de las empresas

\begin{tabular}{|c|c|c|c|c|c|c|c|c|}
\hline Competencias & & Media & Mediana & Moda & $\begin{array}{l}\text { Desv. } \\
\text { típica }\end{array}$ & Mín. & Máx. & $\begin{array}{c}\text { Lambda } \\
\text { de Wilks } \\
\text { p-valor }\end{array}$ \\
\hline \multirow{2}{*}{ Capacidad técnica } & $\mathrm{AL}$ & 4.398 & 5 & 5 & 0.755 & 1 & 5 & \multirow{2}{*}{$.002^{*}$} \\
\hline & TE & 4.553 & 5 & 5 & 0.605 & 3 & 5 & \\
\hline \multirow{2}{*}{$\begin{array}{l}\text { Administración de los } \\
\text { trabajos }\end{array}$} & $\mathrm{AL}$ & 4.550 & 5 & 5 & 0.691 & 1 & 5 & \multirow{2}{*}{$.049 *$} \\
\hline & TE & 4.632 & 5 & 5 & 0.561 & 2 & 5 & \\
\hline \multirow{2}{*}{$\begin{array}{l}\text { Habilidades de } \\
\text { comunicación oral y escrita }\end{array}$} & $\mathrm{AL}$ & 4.474 & 5 & 5 & 0.748 & 1 & 5 & \multirow{2}{*}{.119} \\
\hline & $\mathrm{TE}$ & 4.554 & 5 & 5 & 0.669 & 2 & 5 & \\
\hline \multirow{2}{*}{ Creatividad } & $\mathrm{AL}$ & 3.959 & 4 & 5 & 0.986 & 1 & 5 & \multirow{2}{*}{$.000^{* *}$} \\
\hline & TE & 4.422 & 5 & 5 & 0.700 & 1 & 5 & \\
\hline \multirow{2}{*}{ Iniciativa } & $\mathrm{AL}$ & 4.342 & 5 & 5 & 0.928 & 1 & 5 & \multirow{2}{*}{$.001^{* *}$} \\
\hline & TE & 4.531 & 5 & 5 & 0.682 & 1 & 5 & \\
\hline \multirow{2}{*}{ Motivación } & $\mathrm{AL}$ & 4.549 & 5 & 5 & 0.799 & 1 & 5 & \multirow{2}{*}{$.000^{* *}$} \\
\hline & TE & 4.746 & 5 & 5 & 0.535 & 2 & 5 & \\
\hline \multirow{2}{*}{ Trabajo en equipo } & $\mathrm{AL}$ & 4.598 & 5 & 5 & 0.819 & 1 & 5 & \multirow{2}{*}{$.001^{* *}$} \\
\hline & $\mathrm{TE}$ & 4.755 & 5 & 5 & 0.509 & 2 & 5 & \\
\hline
\end{tabular}

AL: alumno; TE: tutor empresa

* indica un nivel de confianza superior al 95\%. ${ }^{* *}$ indica un nivel de confianza superior al 99\%

2) Competencias evaluadas por los alumnos y por los tutores en las empresas según el sexo.

En este apartado trataremos de mostrar la relación de importancia otorgada por los alumnos y por los tutores a las competencias desarrolladas en las prácticas teniendo en cuenta el sexo de los alumnos. Así, en la Tabla 4, podemos apreciar que:

- Desde el ámbito del alumnado, tanto los alumnos del sexo femenino como masculino otorgan la puntuación media más alta al trabajo en equipo, siendo de 4.571 para los hombres y de 4.608 para el caso de las mujeres. La puntuación 
media más baja dada por cualquiera de los sexos es para la creatividad, 3.921 en los hombres y 3.974 para las mujeres. La mayor diferencia observada entre las puntuaciones promedio dadas por hombres y mujeres para cada una de las competencias corresponde a la iniciativa, siendo de 0.237.

- Desde el ámbito de los tutores, éstos conceden la puntuación media más alta en el caso de los alumnos hombres al trabajo en equipo, $4.766 \mathrm{y}$, en el caso de las mujeres a la motivación, 4.795. La puntuación media más baja dada por los tutores tanto al alumnado masculino como femenino es en creatividad, $4.377 \mathrm{y}$ 4.440, respectivamente. La mayor diferencia observada en la puntuación promedio entre hombres y mujeres corresponde a la motivación siendo del 0.171.

- Si realizamos una comparación entre las puntuaciones promedio ofrecidas por los alumnos y por los tutores, en todos los casos los tutores dan puntuaciones medias más elevadas. Y es en creatividad donde encontraremos una mayor variabilidad entre la puntación otorgada por los tutores y por los alumnos, para cualquiera de los sexos.

Tabla 4

Puntuación promedio de las competencias desagregada por sexo

\begin{tabular}{lccc}
\hline Competencias & & Hombres & Mujeres \\
\hline \multirow{2}{*}{ Capacidad técnica } & AL & 4.390 & 4.401 \\
& TE & 4.519 & 4.566 \\
\hline \multirow{2}{*}{ Habilinistración de los trabajos } & AL & 4.487 & 4.574 \\
& TE & 4.532 & 4.672 \\
\hline \multirow{2}{*}{ Creatividad } & AL & 4.429 & 4.492 \\
& TE & 4.474 & 4.585 \\
\hline \multirow{2}{*}{ Iniciativa } & AL & 3.921 & 3.974 \\
\hline \multirow{2}{*}{ Motivación } & TE & 4.377 & 4.440 \\
\hline \multirow{2}{*}{ Trabajo en equipo } & AL & 4.171 & 4.408 \\
& TE & 4.468 & 4.557 \\
\hline
\end{tabular}

AL: alumno; TE: tutor empresa

3) Competencias evaluadas por los alumnos y por los tutores en las empresas según la titulación

Teniendo en cuenta la titulación de donde proceden los alumnos que realizan las prácticas (ADE o Turismo), en la Tabla 5 podemos observar la valoración dada a las competencias desarrolladas en las prácticas por sus alumnos y por los tutores. 
- Desde el ámbito del alumnado, los alumnos de ADE otorgan la puntuación media más alta a la administración de trabajos, 4.538 y los alumnos de Turismo al trabajo en equipo, 4.737. Para los alumnos de las dos titulaciones, se pone de manifiesto que, de las 7 competencias analizadas la que menos puntación media recibe es la creatividad, 3.892 en ADE y 4.054 en Turismo. La mayor diferencia observada entre las puntuaciones promedio dadas en las dos titulaciones corresponde a la iniciativa, siendo de 0.333 .

- Desde el ámbito de los tutores, la competencia con puntuación más alta en ADE es la motivación, 4.796 y para Turismo es el trabajo en equipo, 4.796. Al igual que los alumnos, los tutores confieren la menor puntuación promedio a la creatividad, 4.436 en ADE y 4.404 en Turismo. La mayor diferencia observada en la puntuación promedio entre ADE y Turismo corresponde a la motivación siendo del 0.118 .

- Si realizamos una comparación entre las puntuaciones promedio ofrecidas por los alumnos y por los tutores, en todas las competencias los tutores dan puntuaciones medias más altas. Y es en creatividad donde apreciamos una mayor variabilidad entre la puntuación otorgada por los tutores y por los alumnos, para cualquiera de las titulaciones.

Tabla 5

Puntuación promedio de las competencias según titulación

\begin{tabular}{lccc}
\hline Competencias & & ADE & Turismo \\
\hline \multirow{2}{*}{ Capacidad técnica } & $\mathrm{AL}$ & 4.403 & 4.391 \\
& TE & 4.595 & 4.496 \\
\multirow{2}{*}{ Administración de los trabajos } & $\mathrm{AL}$ & 4.538 & 4.565 \\
& $\mathrm{TE}$ & 4.627 & 4.640 \\
\hline \multirow{2}{*}{ Habilidades de comunicación oral y escrita } & $\mathrm{AL}$ & 4.456 & 4.500 \\
& $\mathrm{TE}$ & 4.582 & 4.513 \\
\hline \multirow{2}{*}{ Creatividad } & $\mathrm{AL}$ & 3.892 & 4.054 \\
\multirow{2}{*}{ Iniciativa } & $\mathrm{TE}$ & 4.436 & 4.404 \\
\hline \multirow{2}{*}{ Motivación } & $\mathrm{AL}$ & 4.203 & 4.535 \\
& $\mathrm{TE}$ & 4.516 & 4.553 \\
\hline \multirow{2}{*}{ Trabajo en equipo } & $\mathrm{AL}$ & 4.532 & 4.574 \\
& $\mathrm{TE}$ & 4.796 & 4.678 \\
\hline
\end{tabular}

AL: alumno; TE: tutor empresa

\section{Conclusiones}

La realización de prácticas externas en empresas proporciona un entorno ideal para el desarrollo y evaluación de las competencias profesionales que deben adquirir 
los alumnos. Al alumnado les permitirá tanto aplicar las capacidades y conocimientos adquiridos en sus estudios como reforzar la adquisición de estos conocimientos, de las habilidades profesionales y de las actitudes. Las empresas podrán comprobar si las competencias desarrolladas por los alumnos se adaptan a las requeridas en los puestos de trabajo y a las universidades les permitirá conocer si su formación se adapta a las exigencias del mercado laboral, que en la actualidad presta especial atención a la creatividad, competencia íntimamente relacionadas con la capacidad de innovar y el éxito en el emprendimiento. Teniendo en cuenta estas premisas el objetivo de este trabajo es analizar en qué medida existe una correspondencia entre la formación académica recibida por el alumnado y las exigencias del mercado laboral desde punto de vista competencial, con especial atención a la creatividad.

Basándonos en nuestros grupos de referencia, alumnado y tutores en las empresas, los resultados obtenidos en este estudio muestran que en general, la puntuación promedio de todas las competencias evaluadas por ambos es satisfactoria, pues en todos los casos es alta y superior a 4 sobre 5, debiendo exceptuar de estos resultados a la creatividad, competencia que ha sido evaluada como la más baja. Además, las puntuaciones dadas por los tutores en las empresas para cualquiera de las competencias analizadas son siempre más elevadas que las calificadas por los alumnos. Esto último nos puede llevar a sospechar que tal vez los tutores pueden estar siendo excesivamente complacientes en las altas valoraciones otorgadas, si bien según los resultados obtenidos entendemos son perfectamente válidas ya que discriminan que formación competencial resulta más adecuada o más limitada.

Teniendo en cuenta el análisis de las competencias según el género y la titulación que cursan los alumnos, las conclusiones más relevantes son:

- El análisis de las competencias según el sexo nos ha permitido observar que desde el ámbito de los tutores, éstos otorgan la puntuación media más alta, para los alumnos hombres al trabajo en equipo y, en el caso de las mujeres a la motivación. Desde el punto de vista del alumnado, los alumnos tanto del sexo femenino como masculino otorgan la puntuación media más alta al trabajo en equipo. La puntuación media más baja para ambos sexos y, tanto desde la perspectiva de los tutores como del alumnado, es en creatividad.

- Teniendo en cuenta la titulación que cursan los alumnos, ADE o Turismo, tanto desde la perspectiva de los tutores como del alumnado, la competencia con puntuación más alta en ADE es la motivación y para Turismo el trabajo en equipo. La puntuación media más baja en ambas titulaciones $\mathrm{y}$, tanto desde la perspectiva de los tutores como del alumnado, al igual que ocurría en el análisis por sexo, vuelve a ser la creatividad.

\section{Discusión y aplicabilidad de los resultados}

En cuanto a las implicaciones que tiene este trabajo, si bien los datos obtenidos pueden ser considerados de interés tanto para los alumnos como para los responsables de prácticas y para los empleadores, creemos que en el mundo universitario y concretamente para los docentes es donde más implicaciones tendrían, sobre todo con 
el objetivo de mejorar o potenciar competencias tales como la creatividad, que como hemos podido observar es la peor valorada tanto por el alumnado como por los tutores en las empresas. En este sentido, el proyecto Tuning (2007) reconoce la creatividad como una competencia genérica a desarrollar por todos los estudiantes universitarios, independientemente de la rama de estudios que cursen, pero cuya inclusión entre las competencias a desarrollar en los grados españoles es inferior a lo deseable. El escaso desarrollo de la creatividad en los estudios universitarios también ha sido puesto de manifiesto en otros países por investigadores tales como Cheung, Rudowicz, Xiao y Kwan (2003), Rinaudo y Donolo (1999) o Soler (2003).

Además de su positivo efecto en el ámbito empresarial consolidado, no podemos olvidar que la creatividad está íntimamente relacionada con la innovación y el emprendimiento por lo que fomentar la capacidad y pulsión para crear nuevos negocios e innovar es especialmente importante en el contexto económico y social actual.

La Universidad debe fomentar la educación emprendedora pues es un elemento clave para la mejora de la empleabilidad de los estudiantes. Las Universidades deben transmitir a sus alumnos además de conocimientos y ciencia, valores como la creatividad, la iniciativa, la innovación, la responsabilidad, etc. que contribuyan a desarrollar el potencial emprendedor de los estudiantes. Una vez detectada esta carencia, la siguiente pregunta que hemos de plantearnos es si desde los centros de formación universitaria estamos haciendo lo necesario y, de ser la respuesta negativa, si es posible subsanar esta limitación. El aspecto positivo es que existen numerosas evidencias que ponen de manifiesto que es posible fomentar la creatividad desde las aulas (Runco, 2014).

Siguiendo a Runco (2014), es importante destacar que es fundamental promover una actitud positiva hacia la creatividad (personas e ideas) y apoyar al estudiante de forma incondicional, motivándolo con el fin de fortalecer su ego, seguridad y confianza, dentro de una atmósfera y contexto físico positivo, permisivo y constructivo, eliminado las posibles barreras e inhibiciones aunque con prudencia, sabiendo cuándo es necesario seguir las reglas y el comportamiento socialmente aceptable.

Respecto al trabajo a desarrollar dentro de este clima propicio, se enfatiza la necesidad de que se realice con esfuerzo, ética y constancia y las tareas y actividades fomenten el pensamiento divergente, la detección (además de la solución) de problemas, el trabajo independiente y personalizado y la generalización, aplicando lo aprendido a contextos reales.

Metodológicamente, es importante medir la información que se le ofrece al alumno, que ha de ser cuantitativamente equilibrada, y marcar unos objetivos manejables y específicos. Es importante hacer entender al alumno cuándo ha de basarse en la memoria y cuando ser original. La intervención del profesor ha de ser cada vez menor, reduciendo paulatinamente su ayuda e incrementando la dificultad y exigencia de las tareas, aportando feedback inmediato al trabajo realizado.

Es igualmente importante fomentar la implicación en actividades extracurriculares ya que constituyen un poderoso contexto para la creatividad y el desarrollo del talento.

En el caso específico de las titulaciones objeto de estudio en las universidades españolas, como hemos señalado, son pocos los programas que otorgan a la creatividad un papel prominente y ni siquiera se mencionan en muchas titulaciones de ADE y Turismo entre las competencias a desarrollar. 
Esta problemática es también puesta de manifiesto en Estados Unidos por Schlee y Harich (2014) quienes destacan la importancia de la conexión entre creatividad y pensamiento innovador y la necesidad de incluir esta temática en el programa académico, siguiendo la recomendación del informe de la Association to Advance Collegiate Schools of Business (AACSB) sobre la conveniencia de que los centros de formación fomenten la adquisición de destrezas de resolución de problemas creativos con el fin de incrementar la innovación.

Dada su importancia, recientes estudios (Fekula, 2011; Kerr \& Lloyd, 2008a; Schmidt-Wilk, 2011) coinciden en que la creatividad debería incluirse en los programas de estudios empresariales, necesidad reforzada por el hecho de que en trabajos comparativos realizados, los estudiantes de empresas puntúan más bajo que estudiantes de otras disciplinas (McIntyre, Hite \& Rickard, 2003; Wang, Peck \& Chern, 2010).

En la misma línea, tras una exhaustiva revisión crítica sobre la importancia de la creatividad y la innovación en la empresa en la actualidad, Ghosh (2014) defiende la necesidad de introducirla en el curriculum académico universitario "creativity development of students becomes a priority in business schools" (p. 172) y propone las líneas a seguir para lograr este objetivo, que ha de contar necesariamente con la consideración de todos los agentes implicados: "empresa-industria-facultad-institutoestudiante-sociedad" (p. 176).

Otros autores como Kerr y Lloyd (2008b) proponen sugerentes líneas de acción concretas, basadas en las artes, el proceso creativo y el trabajo en equipo para desarrollar la creatividad en los futuros directivos.

Epstein et al. (2013) destacan que los ejecutivos que tuvieron formación obtuvieron mejores resultados. En definitiva, dar oportunidades y estímulos a los estudiantes para desarrollar el pensamiento creativo, ya que parece evidenciarse que desde las aulas estamos, sin ser conscientes, estableciendo límites y barreras para que el alumnado pueda desarrollar todo su potencial en lo que a su creatividad se refiere.

Detectada esta carencia de la creatividad, en futuras investigaciones, se puede abordar el estudio de esta competencia desde el punto de visa de la enseñanza, donde cabría analizar si se está haciendo desde las aulas de los centros de formación universitaria lo necesario para fomentar esta competencia. Y por otra parte, dados los resultados obtenidos desde el punto de vista de género, donde la valoración dada tanto por tutores como por alumnos a las mujeres es proporcionalmente más baja que la de los hombres, se puede indagar si existe algún fundamento psicológico, neurológico o social como afirman algunos estudios (Abraham, 2016).

\section{Referencias}

Abad, I.M., \& Castillo, A.M. (2004). Desarrollo de competencias directivas. Ajuste de la formación universitaria a la realidad empresarial. Boletín Económico de ICE, 2795, 29-41. Abraham, A. (2016). Gender and creativity; An overview of psychological and neuroscientific literature. Brain Imaging and Behavior, 10(2), 609-618. doi: https:// doi.org/10.1007/s11682-015-9410-8

Agencia Nacional de Evaluación de la Calidad y Acreditación [ANECA] (2005). Libro Blanco Título de Grado en Economía y Empresa. Madrid: ANECA. 
Blanco, F.J., \& Latorre, MaJ. (2012). La enseñanza práctica y pre-profesional en el marco de las Ciencias Administrativas. Revista Innovar, 22(45), 69-82.

Biedma, E., Gómez Aguilar, N., \& Ruiz Barbadillo, E. (2011). El practicum como herramienta de evaluación de las competencias profesionales de los alumnos del Máster de Contabilidad y Auditoría. Revista de Educación en Contabilidad, Finanzas y Administración de Empresas, 2, 113-143.

Boyatzis, R.E. (1982). The Competent Manager: A Model for Effective Performance. New York (EEUU): Riley.

Cheung, C., Rudowicz, E., Xiao, Y., \& Kwan, A. (2003). Creativity of university students: what is the impact of field and year of study?. Journal of Creative Behaviour, 37(1), 42-63.

Climént, J.B. (2010). Reflexiones sobre la educación basada en competencias. Revista Complutense de Educación, 21(1), 91-106.

Delgado, A.Ma. (Coord.), Borges Bravo, R., García Albero, J., Oliver Cuello, R., \& Salomón Sancho, L. (2005). Competencias y diseño de la evaluación continua y final en el Espacio Europeo de Educación Superior. Programa de estudios y análisis. Madrid: Dirección General de Universidades, MEC.

Epstein, R., Kaminaka, K., Phan, V., \& Uda, R. (2013). How is Creativity Best Managed? Some Empirical and Theoretical Guidelines. Creativity and Innovation Management, 22(4), 359-374.

Fernández, G. (2005). Las competencias: clave para una gestión integrada de los recursos humanos. Barcelona: Ediciones Deusto.

Fekula, M. (2011). Managerial creativity, critical thinking, and emotional intelligence: Convergence in course design. Business Education Innovation Journal, 3, 92-102.

Finkel, L., Parra, P., \& Roquero, E. (julio, 2010). La evaluación por competencias: una propuesta metodológica para las prácticas externas de máster oficial en el área de Ciencias Sociales. En T. Hernández, Sociología y sociedad en España. Hace treinta años, dentro de treinta años. Ponencia realizada en X Congreso Español de Sociología, Pamplona.

Freire, M., Teijeiroz, M., \& Pais, C. (2013). La adecuación entre las Competencias adquiridas por los graduados y las requeridas por los empresarios. Revista de Educación, 362, 13-41.

García Manjón, J.V., \& Pérez López, M.C. (2008). Espacio Europeo de Educación Superior, competencias profesionales y empleabilidad. Revista Iberoamericana de Educación, 46(9), 1-12.

Ghosh, K. (2014). Creativity in Business Schools: Towards a Need Based Developmental Approach. Global Journal of Flexible Systems Management, 15(2), 169-178.

Gil, J. (2007). La evaluación de competencias laborales. Educación XX1, 10, 83-106.

Gliem, J., y Gliem, R. (2003). Calculating, Interpreting, and Reporting Cronbach's Alpha Reliability Coefficient for Likert-Type Scales. Conference in Adult, Continuing and Community Education. Midwest Research to Practice. The Ohio State University, Columbus. Recuperado de: http://www.ssnpstudents.com/wp/wp-content/ uploads/2015/02/Gliem-Gliem.pdf

González Lorente, C., \& Martínez Clares, P. (2016). Expectativas de futuro laboral del universitario de hoy: un estudio internacional. Revista de Investigación Educativa, 34 (1), 167-183. 
Hartog, J. (1992). Capabilities, Allocation and Earnings. Boston (EEUU): Kluwer.

Jurado, F. (2009). El enfoque sobre competencias: Una perspectiva crítica para la educación. Revista Complutense de Educación, 20(2), 343-354.

Kerr, C., \& Lloyd, C. (2008a). Pedagogical learnings for management education: Developing creativity and innovation. Journal of Management and Organization, 14, 486-503.

Kerr, C., \& Lloyd, C. (2008b). Developing creativity and innovation in management education: an artful event for transformative learning. En I. King, C. Watkins, N. Nissley, \& C. Funk (Orgs.), Proceedings of the Fourth Art of Management and Organisation Conference. Alberta (Canada): The Art f management \& Organization. Recuperado de http://eprints.qut.edu.au/35677/1/c35677.pdf

Ley Orgánica 5/2002 de 19 de junio de las Cualificaciones y de la Formación Profesional. Boletín Oficial del Estado, 21 de junio de 2002, 147.

Martínez Ruiz, M.A., \& Sauleda, N. (2005). Las universidades ante la necesidad dual de cambio y estabilidad. En M. J. Frau \& N. Sauleda (Edits.), Investigar en diseño curricular. Redes de docencia en el Espacio Europeo de Educación Superior (vol. II, pp.522). Alicante: Editorial Marfil.

Marzo, M., Pedraga M., \& Rivera, P. (2006). Definición y validación de las competencias de los graduados universitarios. Revista de la Educación Superior, 25(4), 140, 49-70.

McIntyre, F.S., Hite, R.E., \& Rickard, M.K. (2003). Individual characteristics and creativity in the marketing classroom: Exploratory insights. Journal of Marketing Education, 25, 143-149.

Mertens, L. (1996). Labour Competence: Emergence, Analytical Frameworks and Institutional Models. Montevideo (Uruguay): Cinterfor/ILO.

Molina, E., Bolívar, A., \& Burgos, A. (2007). Mejorar el practicum de pedagogía. Aportaciones desde la investigación. Revista de investigación educativa, 25(2), 443-461.

Montoro, E.M플, Mora, M., \& Ortiz de Urbina, M. (2012). Análisis de las competencias adquiridas en los estudios de Dirección de Empresas y su grado de aplicación en las prácticas en empresas. Revista Complutense de Educación, 23(1), 241-263.

Oliveros, L. (2006). Identificación de competencias: Una estrategia para la formación en el Espacio Europeo de Educación Superior. Revista Complutense de Educación, 17(1), 101-118.

Proyecto Tuning (2007). Tuning Education Structures in Europe. Informe final. Bilbao: Universidad de Deusto.

Quintana, M.A., Raccoursier, M. S., Sánchez Guzmán, A.X., Sidler, H.W., \& Toirkens, J.R. (2007). Competencias transversales para el aprendizaje en estudiantes universitarios. Revista Iberoamericana de Educación, 44(5), 1-6.

Real Decreto 592/2014, de 11 de julio, por el que se regulan las prácticas académicas externas de los estudiantes universitarios. Boletín Oficial del Estado, 30 de julio de 2014, 184, 60502-60511.

Rinaudo, M.C., \& Donolo, D. (1999). ¿Creatividad en educación? Retos actuales de la enseñanza universitaria. Contextos de Educación, 1(2), 202-219.

Runco, M.A. (2014). Creativity, 2nd Edition: Theories and Themes: Research, Development and Pratice. New York (EEUU): Elsevier, Academic Press. 
Schlee, R.P., \& Harich, K.R. (2014). Teaching creativity to business students: How well are we doing? Journal of Education for Business, 89(3),133-141

Schmidt-Wilk, J. (2011). Fostering management students' creativity. Journal of Management Education, 35, 775-778.

Sijtsma, K. (2009). On the use, the misuse and the very limited of the Cronbach's alpha. Psychometrika, 74(1), 107-120.

Solanes, P., Núñez Núñez, R., \& Rodríguez Marín, J. (2008). Elaboración de un cuestionario para la evaluación de competencias genéricas en estudiantes universitarios. Apuntes de Psicología, 26(1), 35-49.

Soler, M.I. (2003). Creatividad en el ámbito universitario: la experiencia de Chile. Creatividad y Sociedad, 3, 39-45.

Tejada, J., \& Navío, A. (2005). El desarrollo y la gestión de competencias profesionales: Una mirada desde la formación. Revista Iberoamericana de Educación, 37(2), 14-28.

Wang, S., Peck, K.L., \& Chern, J. (2010). Difference in time influencing creativity performance between design and management majors. International Journal of Technology \& Design Education, 20, 77-93.

Zhou, J. (2007). Leading for Creativity: An employee-manger dyadic approach. En T. Davila, M. J. Epstein, \& R. Shelton (Eds.), The Creative Personality: Managing innovative organizations and people (vol. 2 Culture, pp.16-36). Westport, CT (EEUU): Praeger Persperctives.

Fecha de recepción: 22 de noviembre de 2016.

Fecha de revisión: 10 de enero de 2017.

Fecha de aceptación: 10 de enero de 2017. 
